\title{
Design of a Fault Tolerant Real-time Storage System for Multimedia Applications*
}

\author{
Richard Muntz, Jose Renato Santos, Frank Fabbrocino \\ UCLA Computer Science Department \\ Multimedia Laboratory \\ $\{$ muntz,santos,frank\}@ cs.ucla.edu
}

\begin{abstract}
We describe the design and implementation of the RIO (Randomized I/O) multimedia object server which manages a set of parallel disks and supports real-time throughput and statistical delay guarantees. The original implementation, on an SMP (Sun Microsystems E4000), has been ported to a cluster of PCs. The new version uses a similar philosophy with respect to allocation of data storage and scheduling of requests. However the parallel "shared nothing" architecture raises all the issues of latency, scalability, etc. that are familiar in parallel and distributed systems. Our requirements are particularly demanding since we expect to support a variety of multimedia applications with varying workload requirements and data reference patterns. $3 D$ interactive applications, video, hypermedia and interactive scientific visualization of terabyte size data sets are among the applications we support. This paper details the design issues we addressed and the results of detailed simulation studies which dictated the design choices made.
\end{abstract}

\section{Introduction}

\subsection{Motivation}

Information systems are used increasingly for storage and retrieval of multimedia data, such as images, video, audio, 3D graphics, etc. These data types are extremely large (gigabytes) and many have real-time delivery requirements. The real-time requirement affects the design of multimedia systems at different levels; data storage, indexing, processor and memory management, data communication, data display, etc. In this paper we concentrate only on storage system issues. Other aspects of multimedia systems are

\footnotetext{
* This research was supported in part by grants from Intel Corp., and NSF grant IRI-9527178. The second author is also with University of Sao Paulo; and his research is partially supported by a fellowship from $\mathrm{CNPq}$ (Brazil)
}

equally important, but are not discussed here. Our assumption is that multimedia objects are too large to fit entirely in main memory and need to be retrieved from disk on demand. Further, parallel disks are required for the bandwidth and storage capacities anticipated for multiple clients and for high performance applications.

The design requirements for our system are summarized as:

- scalable to hundreds of disks

- cost effective use of commodity PCs and communication hardware

- ability to provide statistical guarantee of maximum response time to serve a request for a data block (order of $10^{-6}$ probability of exceeding the delay bound)

- delay bound on the order of a few hundred milliseconds at most

- for cost effectiveness, the last two items must be achieved even with high disk utilization

- a simple interface to admission control, i.e., relatively simple means to characterize prospective sessions' workload

\section{Creating Random Access Patterns}

New multimedia applications, e.g., 3D interactive virtual worlds, have I/O patterns which are much less predictable than conventional video or audio. In a $3 \mathrm{D}$ interactive virtual world application the user navigates through large 3D graphic models at variable speed and along user controlled paths. In order for the display engine to show the current world view, the graphical models of nearby 3D objects need to be continuously retrieved from disk as the user moves. The access pattern to storage objects thus depends on the speeds and paths selected by the user, which makes prediction imperfect at best. 3D virtual world models have been 
used for different applications such as architectural building design[3], urban city models[6], scientific visualization [7][4], etc.; and will be increasingly common in the future.

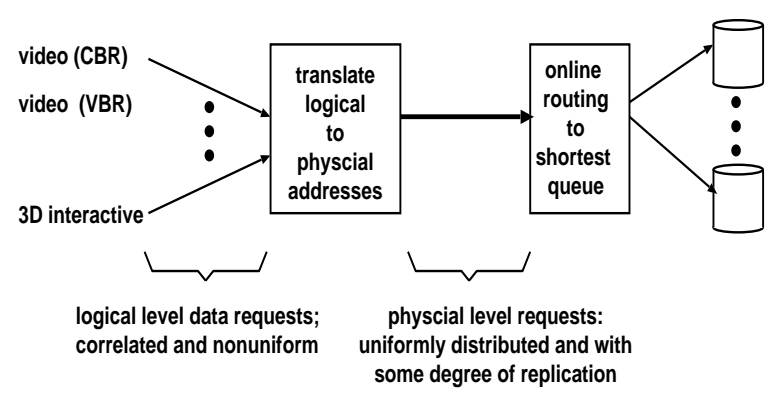

\section{Figure 1. Mapping of all logical level refer- ence patterns to random physical reference patterns and online load balancing based on random replication.}

Not only do applications such as virtual environments produce unpredictable access patterns but we also want to concurrently support multiple user sessions in which different media types are being accessed. Further the fraction of the workload devoted to the different media types will fluctuate with time which adds another dimension to the predictability problem. Our solution is simple: we destroy all access patterns by random allocation of data blocks to the parallel disks! As illustrated in Figure 1 this converts all access patterns to a uniformly random access pattern to the parallel disks. Thus we have only one access pattern to deal with; albeit one that appears to present some difficulties. This may seem to be a rash decision and indeed we expect sometimes to sacrifice some performance but in return we gain predictability. In fact we will show later that there is actually very little sacrifice in performance for our applications; typically less than $10 \%$. For the moment, consider the issue of predictability. It may strike the reader as contradictory to randomly allocate data to obtain a reference pattern with no correlation between data accesses and then claim that this leads to predictability. The apparent paradox is resolved as follows. First, we want predictability, not determinism; so having a known stochastic distribution for the reference pattern is appealing. Having unknown behavior that is a function of what applications will be run (even future applications that do not currently exist) is a worse situation. Secondly, for soft real-time systems with statistical guarantees of delay bounds, we want to have response time distributions with "short tails". With just random allocation of data, the successive requests will be uniformly distributed over the disks and the tail of the response time distribution will be relatively "heavy" (i.e., the time $\tau$, such that only one in a million requests suffer a larger latency than $\tau$ will be larger than desirable.). The "trick" we invoke is to use random replication of data and online load balancing which is known to cause an exponential reduction in the tail of the response time distribution [1][8].

We note that replication for load balancing is not a new idea. In other applications essentially the same idea is presented in terms of tasks that have to be routed to processing nodes[1][8]. Probing of the state (e.g., queue lengths) is done at some randomly chosen set of processing nodes and then the job is routed to the one least loaded. Here we have to randomly replicate data blocks so that when a request is made to access a block, it is equivalent to randomly selecting the storage nodes to probe and route to the least loaded. This mapping from processing task to storage blocks is trivial but our application of the idea in this study is still novel for several reasons:

- we apply the replication and online load balancing to support real-time requirements rather than best-effort, i.e., gains in the mean values of performance metrics

- we consider the application in a parallel system in which clients (that originate requests) do not have a global view of the systems and therefore can not route requests to the least occupied disk. We propose a simple method of implementation that effectively overcomes this lack of global state information and achieve close to optimum performance.

- we consider not only the online scheduling of requests in a nominal system state with all components operational but also consider the tradeoffs between (a) performance in degraded mode (with a failed disk) and (b) alternate way to replicate data to decrease the mean time between data inaccessibility (i.e., time until both copies of some data item is inaccessible).

The original RIO prototype was implemented on a SUN E4000 machine, having 10 Ultrasparc processors, 1.25 Gbytes of shared memory, fourteen 4 Gbyte disks dedicated to multimedia data storage, with ATM and Ethernet connections to client machines. This prototype is operational and has been used to simultaneously support delivery of MPEG encoded videos, and 3D urban simulation city models. Other applications for real-time scientific visualization and medical VR are also under development. A port of the system to a cluster of workstations is underway. This paper describes design issues and summarizes simulation and measurement results with particular emphasis on the faulttolerant issues for this soft real-time system.

\subsection{Organization}

The main body of the paper (Section 2) presents simulation results which are used to explore several major design 
issues. We first show some comparison of simulation results with measurement results which were taken on our current SMP prototype. The results here are mainly to validate the simulation of the disks. Next we describe a range of alternative data layout strategies and evaluate them via simulation. The metrics used are (a) utilization, (b) delay bound, (c) probability of missing the delay bound. Throughout the paper we assume a probability of missing the delay bound is set to $10^{-6}$ so most of the figures show the delay bound that can be achieved as a function of load. We first evaluate a set of data layout schemes assuming a centralized scheduler that has perfect knowledge of the disk queues. We consider both normal operation and with one failed disk. We assume that one disk failure is common enough that the system should be designed to continue operation in this case and maintain all ongoing client sessions with the guaranteed QoS (Quality of Service). We next introduce the issue of imprecise knowledge of the state of the disk queues and consider, via simulation, several approaches to this problem. One scheme which appears to be new, is to "concentrate" client requests in relatively few "router" nodes which then route requests to storage nodes. The router nodes each independently do their best to balance the load that they manage over the disks. The routers do this without any direct state information from the storage nodes; rather they only use knowledge of where they recently sent disk block requests. Simulations show the degree to which this is effective.

\section{Simulation Results}

\subsection{System Overview}

In this paper, we assume that requests waiting on a particular disk queue are serviced according to an EDF (Earliest Deadline First) policy. For simplicity, we assume that all requests have the same delay bound and thus are serviced in FIFO (First In First Out) order ${ }^{1}$.

The disk performance highly depends on the selected block size that is read in each disk access. Larger block sizes will generate higher disk throughput $(\mathrm{MB} / \mathrm{sec})$ since I/O overhead such as rotational latency, seek time, etc., will be amortized over a longer transfer time. However increasing the block size increases the size of the buffers required for each stream which has an associated cost. Also, having larger block sizes increases the probability of reading superfluous data for applications such as 3D virtual world models

\footnotetext{
${ }^{1}$ In RIO we can also use a modified elevator disk scheduling algorithm which minimizes seek time by reordering requests according to their position on the disk surface. Using the elevator algorithm can slightly increase the disk throughput, but may also increase the delay bound since requests are reordered and not serviced according to their deadlines. Depending on which effect is dominant, real-time performance can be improved or not
}

where data accesses are not sequential and logical data are not generally a multiple of the block size. Therefore, there are multiple tradeoffs when selecting the block size, which have to be kept in mind mind, but determining the optimal block size is beyond the scope of this paper. Here, we assume that a block size is selected at system configuration, and then we use this block size to obtain the disk throughput $(\mathrm{MB} / \mathrm{sec})$ or, equivalently, the disk service rate (req/sec). The disk throughput can be determined experimentally, or using a disk model with performance characteristics from the disk manual.

We define the maximum load supported by the system, $\lambda_{\max }$, as the load (req/sec) that can processed by the system when all disks are working at full capacity, i.e. $\lambda_{\max }$ is equal to the sum of the service rates of all disks. We assume an admission control and a traffic policing modules that guarantee that certain constraints on the disk traffic are met. New sessions specify their traffic requirements to the admission control module, that accepts the new session only if the total aggregate load specified by all sessions, including the new one, satisfy the system constraints. The traffic policing module is responsible for enforcing sessions to satisfy their traffic specifications declared at their admission. In particular, we assume that admission control and traffic policing will guarantee that the total number of disk access in any arbitrary interval of duration $\mathrm{T}$ is less or equal $n_{T}$, where $T$ and $n_{T}$ are system parameters. The details of how admission control and traffic policing can make such guarantees are left as future research. In our simulations and experiments, we divide the time in consecutive intervals $T_{i}$ $(i \geq 0)$, each with duration $T$, and generate a fixed number of requests $n_{T}$ in each interval $T_{i}$. Thus, the load of the system is given by $\lambda=n_{T} / T$ (requests/sec). To ensure that the system is stable we must have $\lambda<\lambda_{\max }$. Moreover, we assume that the $n_{T}$ requests in each interval $T_{i}$ are generated by many independent sessions, and thus we assume their arrival times are randomly distributed over each $T_{i}$.

\subsection{Simulation Validation}

In order to evaluate the performance of the storage system for a larger number of disks than is currently available on the prototype, a simulator was developed and validated.

Unless otherwise stated, all simulations used $128 \mathrm{~KB}$ blocks. For this block size, the disks used in our prototype (SEAGATE Barracuda ST15150W) can support a maximum load of $28.3 \mathrm{req} / \mathrm{sec}$ each, with an average service time of $35.3 \mathrm{~ms}$ per request, assuming requests are serviced in FIFO order. Therefore the simulated system with 64 disks could support a maximum load $\lambda_{\max }=1811 \mathrm{req} / \mathrm{sec}$. The duration $\mathrm{T}$ of each interval $T_{i}$ was set to ten times the average disk service time, i.e. $T=353 \mathrm{~ms}$. However, more 
extensive simulations, not shown here due to space limitations, have demonstrated that performance is relatively insensitive to the duration of the interval $T$.

The results shown in this paper are presented in terms of the delay bound normalized by the mean disk I/O service time, as function of the system utilization $\rho=\lambda / \lambda_{\max }$. The results are normalized, such that they can be extrapolated for other disk service rates and mean service times associated with other block sizes. We observed in our experiments that a simple normal distribution model is a good approximation for the disk I/O service time. Different block sizes, generates service time distributions with different mean and coefficient of variation and thus different delay bound guarantees. For block sizes on the range of $4 \mathrm{~KB}$ to $256 \mathrm{~KB}$, on the disks used in our prototype, we experimentally observed that the relative performance of RIO have only small variations, despite the fact that the service time distributions have different coefficient of variation. Therefore, the results presented here can be used to approximately estimate performance of other block sizes, by just scaling the reported results by the mean service time of other block sizes. However, this is just an approximation and small variations should occur.

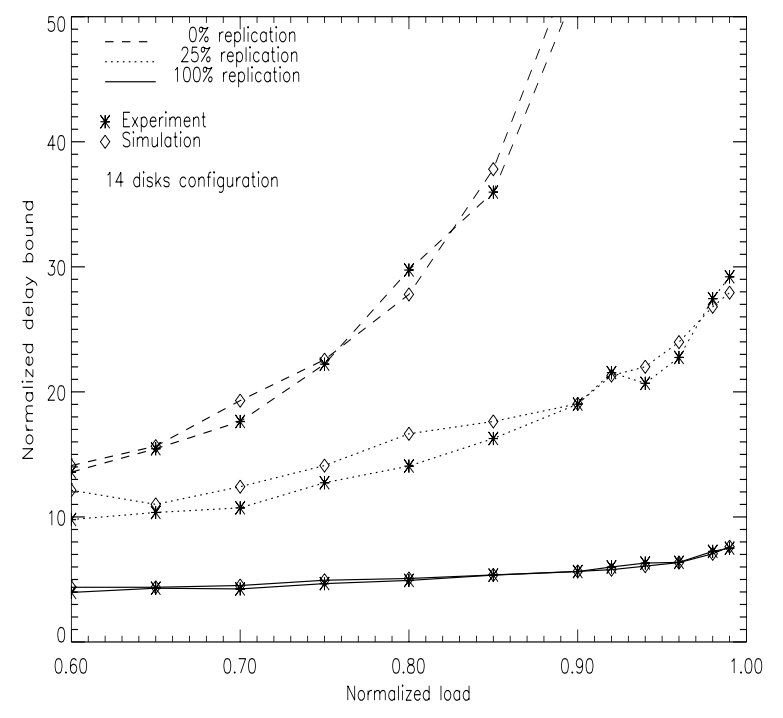

Figure 2. Simulation validation experiments.

In order to validate our simulation we compared the results generated by simulation with the experimental results obtained from measurements on the Sun E4000 prototype with a configuration using 14 disks. Clearly the results of the simulation track very closely the measured results in Figure 2, confirming that our simulation is modeling the disk behavior accurately. Further simulations reported in this paper use the same disk models. It is anticipated that the PC cluster versions may show some differences due to systems software, bus speeds, etc. but these results give us confidence in the simulation being accurate enough for the design comparisons we need to make at this early stage.

In addition to illustrating the accuracy of the simulation, Figure 2 also shows the effectiveness of online load balancing with varying degrees of replication. For example we see that even as little as $25 \%$ replication provides a significant decrease in the delay bound that can be guaranteed with probability $1-10^{-6}$; particularly under relatively high load. (This observation was also made in [8] based on an analytic model of the same type of system we analyze here but with more restrictive assumptions.)

\subsection{Fault tolerance}

The use of partial replication as described in the last sections is an effective method for providing short term load balance among the parallel disks. However, in order to provide tolerance to disk failures we need to consider alternative schemes in which data is still accessible, even with one failed disk. There are basically two approaches; one is to use full replication and the other is to use parity groups such as used in RAID schemes[10].

One possibility is to use RAID technology to provide fault tolerance and partial replication of data blocks to provide short term load balancing. In this approach, the total number of disks in the system, $D$, are divided into $N_{A}$ disk arrays with $D_{A}=D / N_{A}$ disks each. Each disk array is a RAID level 5 [10] with parity group declustering [9] that stores data organized as parity groups of size $G$ distributed on $D_{A}$ different disks $(G-1$ data blocks and 1 parity block; $\left.G \leq D_{A}\right)$. A fraction $\alpha$ of the data blocks are then randomly replicated across all disks of the system. Replicating data blocks across all disks, instead of replicating them only within the same disk array allows balancing the load among disks in the disk array as well as across disk arrays. Parity blocks are not replicated, since they are only used when a disk has failed and, while in that mode, only to reconstruct data blocks that resided on the failed disk. Data blocks are read during both normal conditions and degraded operation. The ratio of useful data space and total storage space for this scheme is given by:

$$
U=\frac{1}{1+\alpha+\frac{1}{G-1}}
$$

Note that this scheme has performance identical to that of a system that does not use RAID, when the system is operating under normal conditions (without failure) with only read operations, since parity groups are only used if a disk fails. Later, after introducing alternative schemes we will analyze performance when a disk fails. 
A second scheme is to use $100 \%$ replication for both load balancing and fault tolerance purposes. Since all data blocks are replicated, with any single disk failure all data is still available, i.e., accessible. However, using random replication across all disks does not support simultaneous failures of any two disks, since every pair of disk contains some data that is stored only on that disk pair. Imposing constraints on where data can be replicated can improve fault tolerance by allowing the system to survive at least some combinations of multiple disk failures. However these same constraints can also reduce the capacity for short term load balancing during both normal operation as well as degraded mode.

One such $100 \%$ replication scheme is chained declustering [5], in which half the data which is stored on a given disk $i$ is replicated in the next logically consecutive disk $i+1$. This scheme is illustrated in Figure 3(b). Chained declustering allows multiple disk failures, provided only that no two failures occur on logically consecutive disks. Chained declustering pays for increased fault tolerance with less flexible short term load balancing as our simulation results will show. A generalization of chained declustering, which we call overlapping clusters, allows the system designer to trade off fault tolerance and real-time performance. In the overlapping clusters scheme each disk can be thought of as two logical volumes, each holding half the storage capacity of a physical disk. Logical volumes are grouped into overlapping clusters of size $C$ and offset by $\mathrm{C} / 2$ with respect to the neighboring clusters as illustrated in Figure 3(a). The two copies of a replicated data block are then constrained to be located within one cluster. This scheme allows multiple disk failures provided that no two failed physical disks imply the failure of two logical volumes in the same cluster. This scheme lies between random replication and chained declustering since it supports more disk failures than random replication and less than chained declustering, while providing more flexibility for short term load balancing than chained declustering but not as much as random replication.

In the next sections we evaluate and compare the performance of the following options with respect to fault tolerance:

- Partial replication for load balancing combined with parity groups for reliability

- Full replication for both reliability and load balancing

- Random replication

- Chained Declustering

- Overlapping Clusters

In section 2.4 we evaluate and compare performance of these designs options, both under normal operations and under one disk failure, on a centralized system that has perfect

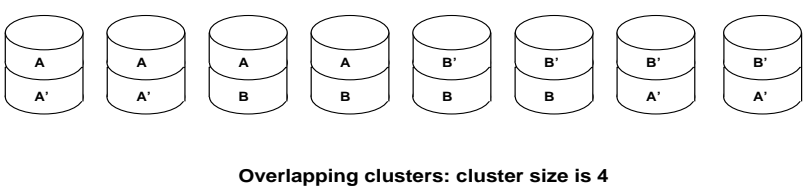

(a)

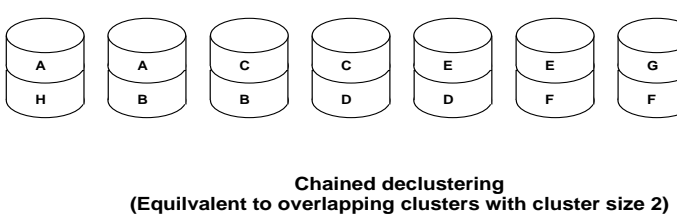

(b)

\section{Figure 3. Replication using overlapping clus- ters; limiting case (cluster size $=2$ ) equiva- lent to chained declustering.}

knowledge of disk queues. In section 2.5 we consider a distributed system, where there is no global knowledge of the current disk queues status.

\subsection{Scheduling with perfect knowledge of disk queue status}

The first set of simulation results compare the data allocation schemes described above both in normal operation and with one failed disk. To separate considerations we assume in this first set of experiments that requests are routed to disks with perfect global knowledge of the disk queues. Later we will consider how scheduling can be done when, due to the distributed nature of the PC cluster, only imprecise knowledge of the disk queues is available.

Unless otherwise stated, all simulations are for systems with 64 disks. When RAID is discussed, the parity group size is 5 , i.e., 4 data blocks and a parity block.

Figure 4 (top) shows the normalized delay bound that can be obtained with the various data layout options. First, the value of random replication is easily seen by considering the three curves that correspond to random replication of $0 \%, 25 \%$ and $100 \%$ of the data. It is clear from these curves that the delay bound that can be guaranteed with probability $1-10^{-6}$ grows considerably with load when there is no replication but is relatively flat when replication is used, even out to $90 \%$ utilization or more. The implication is that, if admission control will allow utilizations up to $90 \%$, then with no replication the delay bound will have to be set at about 50 times the disk service time (e.g., 1.76 sec for $128 \mathrm{~KB}$ blocks); a rather large value. Note that if a lower delay bound is guaranteed to a session when the utilization is lower, then admission control would be held to that guarantee and if further clients requested service, they 

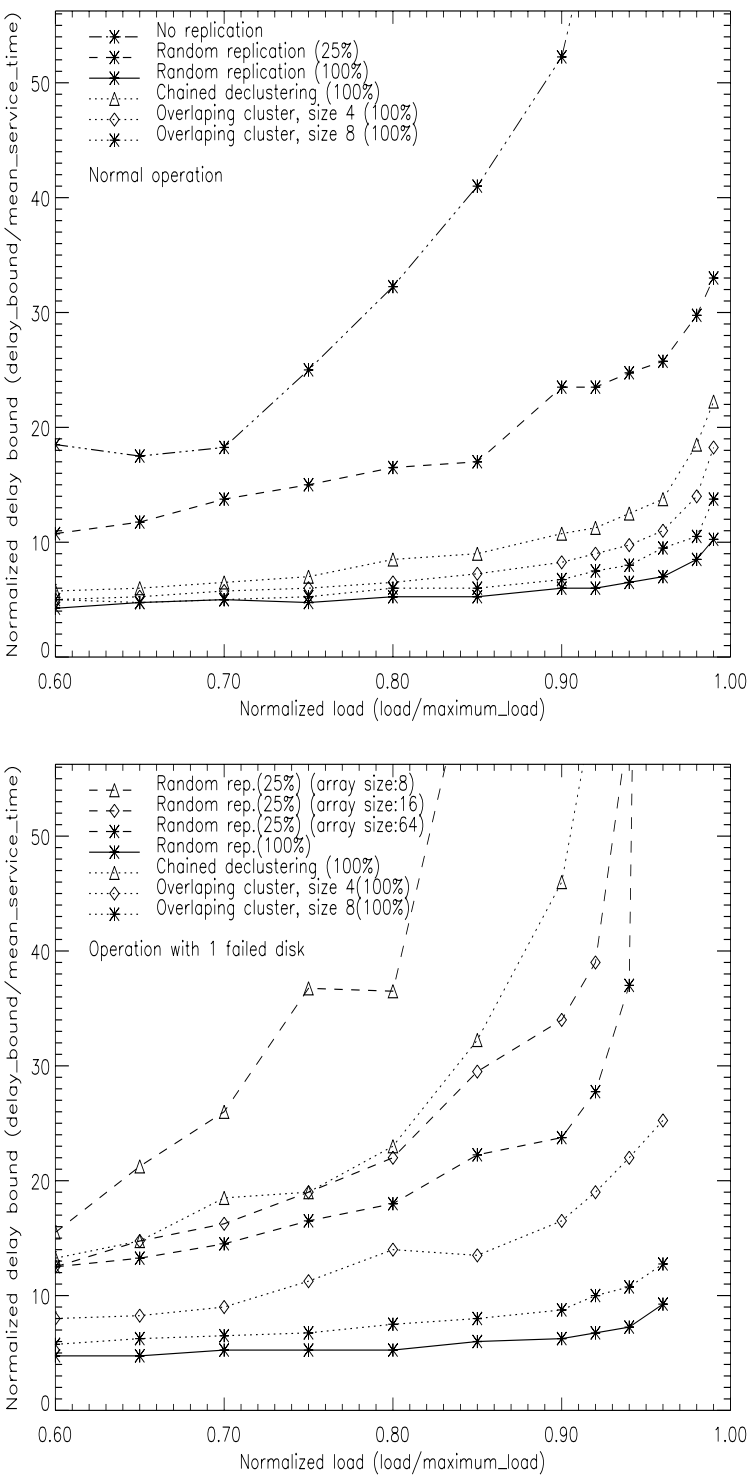

Figure 4. Perfect knowledge online router; normal operation (top) and with one failed disk (bottom).

might have to be denied service in order to meet the delay guarantee made to the earlier session. For this reason we assume that the delay bound is chosen according to the maximum utilization that will be permitted. So, for example, if the largest utilization allowed is $95 \%$, with $100 \%$ random replication we could guarantee a delay bound of about 6 times the average disk service time (e.g., $210 \mathrm{~ms}$ for 128 KB blocks). With $25 \%$ random replication the delay bound would be about 24 times the average disk service time (e.g., $850 \mathrm{~ms}$ for $128 \mathrm{~KB}$ blocks).
Also from Figure 4 (top) we see that chained declustering and the overlapping cluster schemes work reasonably well and, as expected, the larger the clusters are, the more effective it is for short term load balancing and the flatter the curves are for a larger range of utilizations.

Figure 4 (bottom) shows the same type of plot but with one failed disk. In this part of the figure there are a couple of new curves. These correspond to the use of RAID arrays with some additional random replication of data blocks for load balancing. We compare, for example, various size RAID arrays (remember the total number of disks is 64 in all cases), all with $25 \%$ random replication of data blocks. We choose to only look at $25 \%$ replication since, with $G=5$, there is already $25 \%$ overhead for parity blocks and by randomly replicating an additional $25 \%$, the total is already up to $50 \%$. Any further replication would be close enough to $100 \%$ that it would seem simpler and more effective to just use a $100 \%$ replication scheme and be done with it. However, if storage capacity is scarce, the use of RAID with some random replication for short-term load balancing is a possible alternative. (We note that in recent years disk storage capacity is increasing significantly faster than transmission or access rates so that, increasingly disks will be limited by bandwidth as opposed to storage.)

From the results in Figure 4 (bottom) it is clear that the various schemes retain the same relative performance as with no failed disk. However the differences are more pronounced, i.e., the curves separate significantly. Again, if we wanted to allow some maximum utilization and also, not have to drop any ongoing sessions when a disk fails, we can read the delay bound that can be guaranteed from the curves in the figure. So for example, with overlapping clusters of size 9 , and allowing up to $90 \%$ utilization, the delay bound that could be guaranteed (with one chance in a million of missing) is about 8 times the average disk service time (e.g., $320 \mathrm{~ms}$ for $128 \mathrm{~KB}$ blocks). For random replication, the delay bound, under the same circumstances is about 7 (e.g., $250 \mathrm{~ms}$ for $128 \mathrm{~KB}$ blocks). So with the overlapping cluster scheme we have achieved close to the same performance (with and without failure) as with completely random replication. Overlapping clusters also has a somewhat better probability of surviving a second disk failure without loss of data availability. ${ }^{2}$

For reference, Table 1 shows the relative mean time between "data inaccessible" failures (failures where a fraction of data is unaccessible) for all cases. We assume that the mean repair time of disks is much smaller than the mean time between disk failures in the system. If this is the case

\footnotetext{
${ }^{2}$ We do not expect that the system will continue to operate without dropping sessions in the (rare) case of a second disk failure before the first is repaired. However, as long as data does not become inaccessible, the rebuild of a failed disk can be fairly efficient. When data becomes inaccessible from disk, the system must resort to tertiary storage and the rebuild time can be much longer.
} 
the mean rate of "data inaccessible" failures is dominated by double failures, and is proportional to the number of pairs of disks, which if failed, would cause unavailability of data. Table 1 shows the approximate mean time between "data inaccessible" failures relative to a constant $K$ that can be computed as function of the mean time to failure of a disk and the mean time to repair a disk and is the same for all of the systems. The time to data inaccessibility is inversely proportional to the number of disks for which a second failure would cause inaccessibility of some portion of data.

\begin{tabular}{|l|c|}
\hline \multicolumn{1}{|c|}{ Scheme } & $\begin{array}{c}\text { Mean time between } \\
\text { "data inaccessible" } \\
\text { Failures }\end{array}$ \\
\hline Chained declustering & $\frac{K}{2}$ \\
\hline $\begin{array}{l}\text { Random replication (100\%) } \\
\text { (System with D disks) }\end{array}$ & $\frac{K}{D-1}$ \\
\hline Disk array (size 8) & $\frac{K}{7}$ \\
\hline Disk array (size 16) & $\frac{K}{15}$ \\
\hline Disk array (size 64) & $\frac{K}{63}$ \\
\hline Overlapping clusters (size 4) & $\frac{K}{5}$ \\
\hline Overlapping clusters (size 8) & $\frac{K}{11}$ \\
\hline
\end{tabular}

Table 1. Relative mean time between "data inaccessible" failures

\subsection{Decentralized disk scheduling}

Figure 5 illustrates the major factors that we have to deal with in the clustered version of the object server. The number of clients is expected to be large (e.g., hundreds) and the number of storage nodes to be on the order of a few tens. Clients are not expected to directly access storage nodes for several reasons. One is that they should not have to be aware of the details of the replication and load balancing. Also there are potentially so many of them that it would be costly to keep them up to date on the load on the storage nodes, let alone the disks. Our proposed solution is to have a front

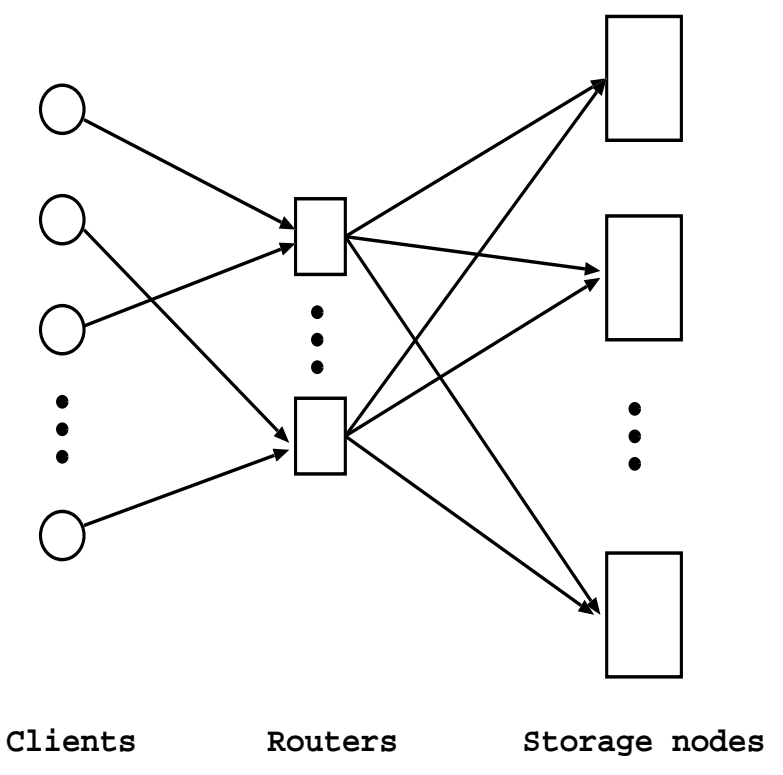

Figure 5. Client requests go through
"routers" which choose replica to be fetched.

end set of nodes that interface to clients. While these nodes will also perform other functions (e.g., admission control, authorization, etc.) we concentrate here on their function in routing client requests to achieve short-term load balancing and thereby, relatively short delay bound guarantee. (Incidentally, when sessions are initiated they are assigned to some router with an aim to approximately equally load the routers.)

The routing function is relatively simple so we expect only between one and ten routers at most. The obvious question is what kind of information the routers will have in order to make routing decisions. In this subsection we present some initial simulation results investigating alternatives.

We use the terms node routing or two level load balancing to mean routing to a storage node to balance the aggregate load at storage nodes. The assumption being that, when there are multiple disks per storage node, the local disk scheduler does routing to the exact disk and uses available replication to do short-term load balancing locally. It should be clear where the two level load balancing terminology comes from. We use the term disk routing or one level load balancing to mean that the router nodes have estimates of disk queues and make the decision as to which disk (when there is a choice) will serve a particular request. We use the term intra-replication, when both copies of a replicated block are stored on disks attached to a single node, and use the term inter-replication otherwise. Thus, node routing is used for inter-replicated data blocks, while disk 
routing is used for intra-replicated data blocks.

In this section we compare performance of random replication and overlapping clusters on a distributed system, composed of multiple PCs interconnected by a high speed network. Overlapping clusters can be implemented in a cluster of PCs as illustrated in Figure 6. For simplicity we assume that the cluster size is equal to the number of disks in each node. In the example of Figure 6 each node has 4 disks and thus the selected cluster size is also 4 . In this configuration half of the clusters are composed of logical volumes on disks attached to a single node (clusters I1, I2, I3, I4), while the other half have their logical volumes on disks attached to two neighboring nodes (clusters $\mathrm{X} 12, \mathrm{X} 23, \mathrm{X} 34, \mathrm{X} 14)$. We have shown elsewhere [2] that an optimal combination of intra and inter replication, that maximizes load balance and thus performance, is approximately $80 \%$ and $20 \%$, respectively. Organizing overlapping clusters as described in Figure 6 has the advantage of generating approximately $75 \%$ intra-replication and 25\% interreplication, which has performance very close to the optimal 80\%/20\% combination described in [2]. On disadvantage of this configuration is that, even though the system is tolerant to single disk failure, it is not tolerant to single node failures, since all disks of a single node becomes inaccessible when a node fails (assuming each disk is connected to a single node, as illustrated by the solid lines in Figure 6). If however we use dual ported disks, each connected to two different nodes as illustrated by the additional dashed lines in Figure 6, the system becomes tolerant to single node failures. During normal operation, only the connections shown as solid lines are used. When a node fails, neighboring nodes take over the disks originally managed by the failed node, using the connections shown as dashed lines. This however has the disadvantage of the additional cost of more expensive dual ported disks and twice as many disk interfaces on the system nodes. This system would also be more complex, since it will have to logically reassign disks to nodes when a node fails. Another alternative would be to construct overlapping clusters that use disks located on different nodes only. Although this scheme would provide reliability under node failure without requiring dual ported disks, it would provide lower performance, both with and without failure, since the system would have $0 \%$ intra-replication, and all load balancing would have to be done using node routing only, which is less effective. We leave the performance study of this last approach as future research, and concentrate here on the case where overlapping clusters are organized as described in Figure 6, using dual ported disks to support tolerance to node failures.

Figure 7 shows a comparison of two things: one is the effect of "two level" versus "one level" routing and the other is random replication versus overlapping clusters. In these simulations we still assume that the router has exact knowl-

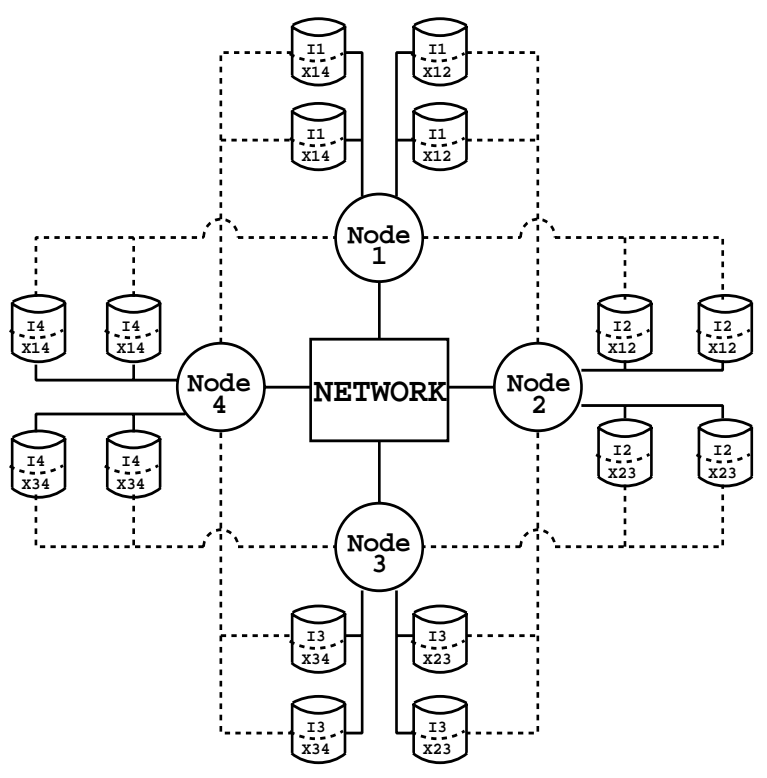

Figure 6. Cluster architecture supporting
overlapping clusters and load balancing in
degraded mode.

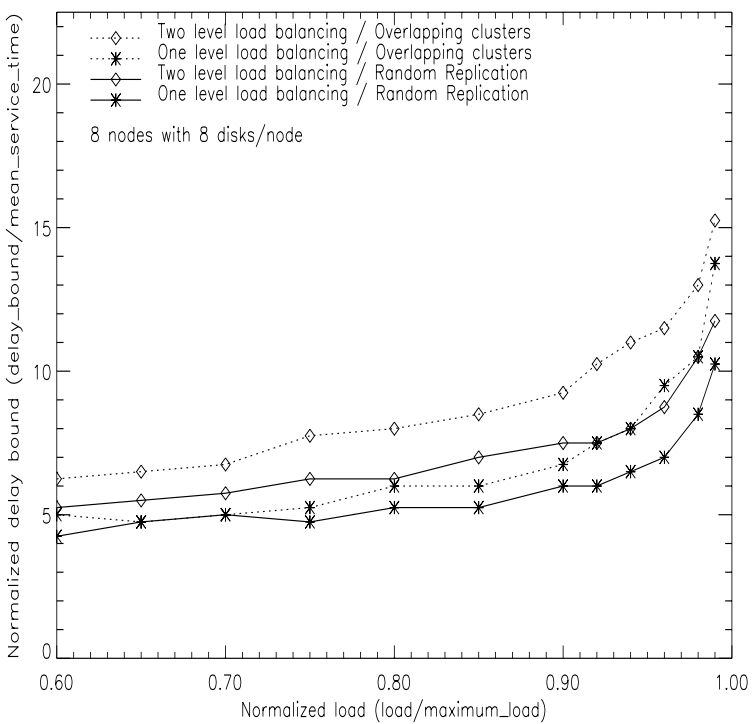

Figure 7. Node (two level) routing vs. Disk (one level) routing: clustered replication vs. random replication.

edge of the the aggregate number of requests queued at a node for two level routing and the number of requests at each individual disk for disk routing. Later we look at a scheme for maintaining approximate state information at 


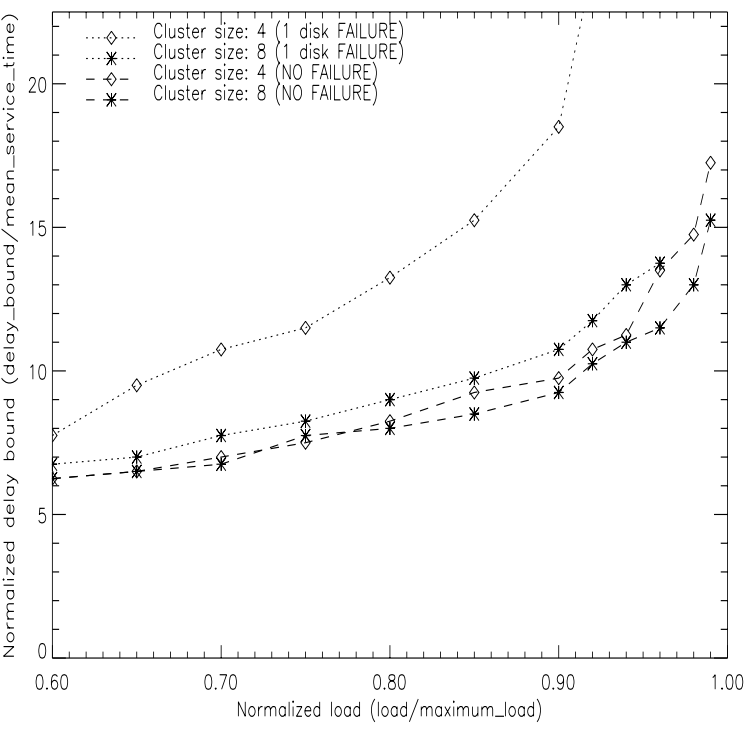

Figure 8. Performance of 64 (top) and 16 (bottom) disk systems with two level routing and different size clusters.

the routers.

Obviously one would expect the two level routing to be less effective than the disk (one level) routing. The results in Figure 7 bear this out. Also the "full" random replication is more effective than the overlapping clusters. So for example, with overlapping clusters (for fault tolerance) and two level scheduling (which may be more practical) the delay bound at $90 \%$ utilization goes from between 5 and 6 times the average disk service time to about 9 or 10 times the average disk service time; not quite double.

Figure 8 illustrates, for two level scheduling, and two different cluster sizes, 4 and 8, the delay bounds that can be achieved with and without a failed disk. We note that, we can guarantee less than 11 times the disk service time as a delay bound, even with a failed disk, if the cluster size is at least 8 . The delay bound that can be achieved is sensitive to cluster sizes below 8 .

\section{A "sliding window" router}

Now we consider what information might be used by the routers to make routing decisions. One possibility is to have the storage nodes periodically send update information about their state to the routers. This raises questions of latency and overhead that are problematic. We decided to explore a different approach. Basically, if the number of routers is small enough such that each handles a not insignificant percentage of the aggregate load, then it may be effective to have each router just balance the load that it imposes on the storage server, i.e., not worry about the load
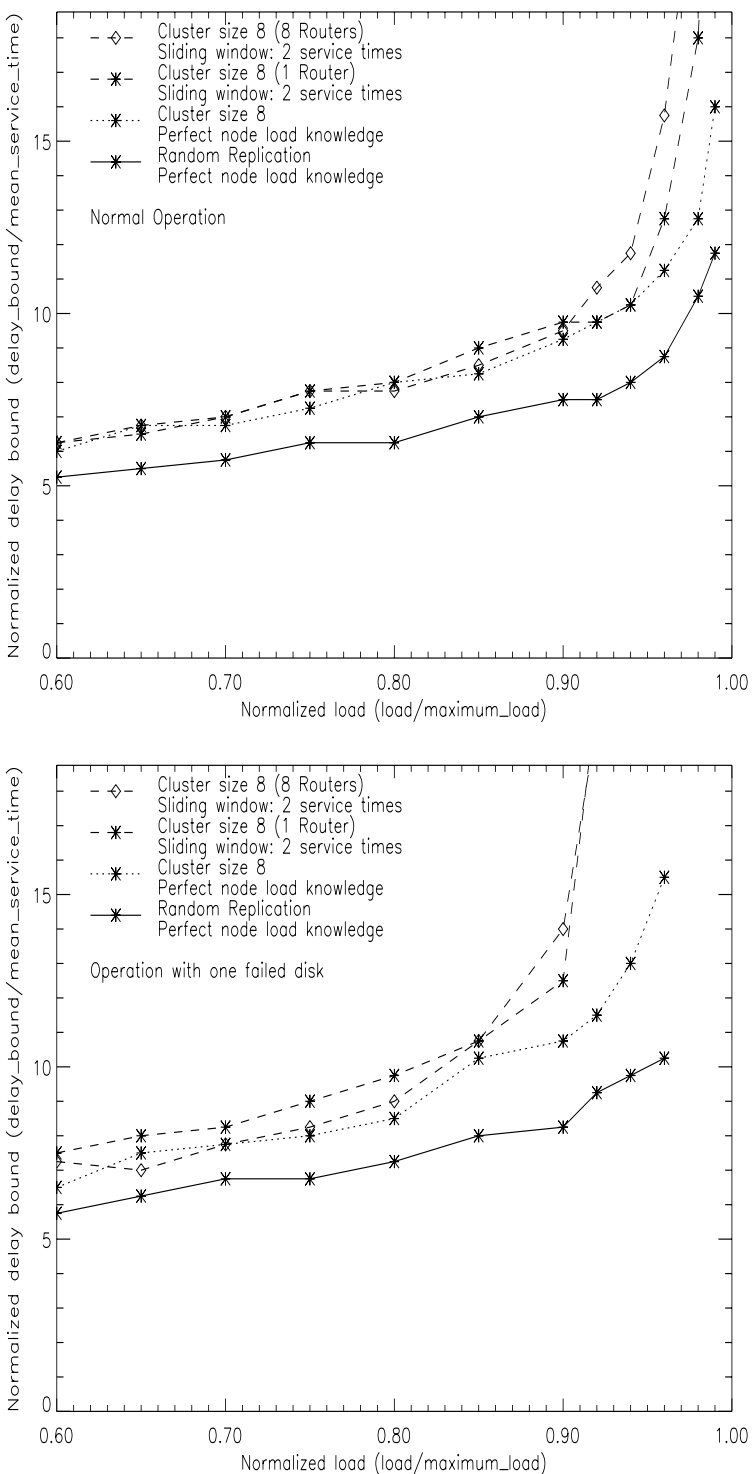

Figure 9. Sliding window scheduler performance.

that other routers place on storage nodes. In this case, perhaps a router could just keep track of the requests it has recently sent to the various storage nodes, use this as an estimate of their current load, and route to the least loaded based on this estimate.

We have carried out simulations of a scheduler based on this idea. A router, in this scheme, keeps track of the number of requests that it has sent to each node during the last $w$ seconds (where $w$ is the window size) and uses this count as the current load on that node. Our results indicate that a relatively "short memory" (i.e., small $w$ ) at the 
router works best. Figure 9 shows a sampling of these results for $w$ equal to two times the average disk service time, which was the window size that produced the better results. The lower curve in each plot is for random replication and perfect knowledge of the state of the disk queues; this is included for reference. From these figures it appears that the sliding window scheduler works almost as well as having precise knowledge of the aggregate load at storage node up to about 85 or $90 \%$ utilization, even when there are several (e.g. 8) routers, each of which tries to balance its own fraction of the load independently of the others.

\section{Conclusion}

We have completed an extensive simulation study of various data layout and scheduling strategies for a clustered, real-time object server. Replication is advocated for both fault tolerance and to achieve a relatively high utilization in combination with a relatively low delay bound guarantee. We evaluated and compared performance of different fault tolerance schemes:

- Partial replication for load balancing combined with parity groups for reliability

- Full replication for both reliability and load balancing

- Random replication

- Chained Declustering

- Overlapping Clusters

Using partial replication combined with parity groups was shown to be the more effective scheme in terms of storage space usage but was shown to have the lowest performance, requiring a lower load than the other schemes to achieve the same delay bound guarantee. Therefore this scheme should be preferred for a system where the scarce resource is data storage as opposed to disk bandwidth. The schemes using full replication support higher loads, but with a higher cost on disk storage and should be used when disk bandwidth is the scarce resource. Among these schemes, random replication was shown to have the best performance, but it was also shown to be the one with lower time between "data inaccessible" failures, since it can not support any double disk failure. On the other hand, chained declustering provides the higher degree of fault tolerance to double disk failures but provides the lowest performance among the $100 \%$ replication schemes. Our Overlapping clusters scheme lies between random replication and chained declustering. Changing the cluster size allows the system designer to trade fault tolerance for performance.

We also proposed a scheme based on independent routers for doing load balancing decisions, and proposed a simple scheme for routing requests to multiple nodes: the sliding window scheduler. Our experiments showed that the performance of the sliding window scheme is very close to the case in which there is perfect knowledge of the nodes load, and also avoids issues of state update propagation to the routers. In summary, the sliding window scheduler was shown to be quite simple and at same time very effective, with the benefit of having no communication overhead.

\section{References}

[1] D.L. Eager, E.D. Lazowska, J. Zahorjan, "Adaptive Load Sharing in Homogeneous Distributed Systems", IEEE Trans. on Software Engineering, pp. 662-675, 1986.

[2] F. Fabbrocino, J.R. Santos, R.R. Muntz, "An Implicitly Scalable, Fully Interactive Multimedia Storage Server “. To appear in the Second International Workshop on Distributed Interactive Simulation and Real Time Applications (DIS-RT '98), Montreal, July 1998

[3] T. Funkhauser, C. Sequin, S. Teller, "Management of Large Amounts of Data in Interactive Building Walkthroughs", ACM SIGGRAPH Proc. of the 1992 Symposium on Interactive 3D Graphics, 1992.

[4] W. Gekelman, D. Leneman, J. Maggs, "Experimental Observation of Alfven Wave Cones", Physics of Plasmas, 1, pp.3775-3783, 1994.

[5] H. Hsiao, D.J. Dewitt, "Chained Declustering: A New Availability Strategy for Multiprocessor Database Machines", Proc. of Data Engineering, pp 456-65, 1990.

[6] W. Jepson, R. Liggett, S. Friedman, "Virtual Modeling of Urban Environments", Presence: Teleoperators and Virtual Environments, Vol. 5, No.1, MIT Press, 1996.

[7] W. Karplus, M.R. Harreld, "The Role of Virtual Environments in Clinical Medicine: Scientific Visualization", Proc. First Jint Conference of International Simulation Societies (CISS), Zurich, Switzerland, pp. 13-17, September 1994.

[8] M.D. Mitzenmacher, "The Power of Two Choices in Randomized Load Balancing", PhD Dissertation, University of California at Berkeley, Computer Science Department, 1996.

[9] R. Muntz, J. C-S. Lui, "Performance Analysis of Disk Arrays Under Failure", Proc. VLDB, Brisbane, Australia, pp. 162173, 1990.

[10] D.A. Patterson, G. Gibson, R.H. Katz, "A Case for Redundant Arrays of Inexpensive Disks (RAID)", SIGMOD 88, pp.109-116, 1988. 